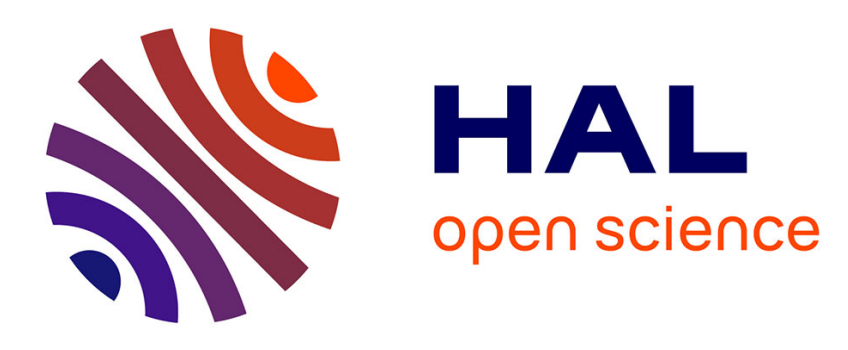

\title{
Propriétés supraconductrices des alliages Al-Ag dans l'état de pré-précipitation
}

\author{
J. Blanc, J.C. Solecki, A. Nemoz
}

\section{To cite this version:}

J. Blanc, J.C. Solecki, A. Nemoz. Propriétés supraconductrices des alliages Al-Ag dans l'état de pré-précipitation. Revue de Physique Appliquée, 1971, 6 (4), pp.551-554. 10.1051/rphysap:0197100604055100 . jpa-00243587

\section{HAL Id: jpa-00243587 https://hal.science/jpa-00243587}

Submitted on 1 Jan 1971

HAL is a multi-disciplinary open access archive for the deposit and dissemination of scientific research documents, whether they are published or not. The documents may come from teaching and research institutions in France or abroad, or from public or private research centers.
L'archive ouverte pluridisciplinaire HAL, est destinée au dépôt et à la diffusion de documents scientifiques de niveau recherche, publiés ou non, émanant des établissements d'enseignement et de recherche français ou étrangers, des laboratoires publics ou privés. 


\title{
PROPRIÉTÉS SUPRACONDUCTRICES DES ALLIAGES Al-Ag DANS L'ÉTAT DE PRÉ-PRÉCIPITATION
}

\author{
J. BLANC, J. C. SOLECKI et A. NEMOZ \\ Centre de Recherches sur les très basses températures \\ Cédex 166, 38-Grenoble
}

\begin{abstract}
Résumé. - Pour expliquer le comportement des propriétés supraconductrices des alliages $\mathrm{Al}-\mathrm{Ag}$ dans l'état de ségrégation en zones Guinier-Preston, nous proposons un modèle basé sur les effets de proximité entre phases normale et supraconductrice. Les résultats sont en accord avec l'hypothèse de l'existence d'une lacune de miscibilité contrôlant la croissance des zones.

Abstract. - To explain the behaviour of the superconductive properties of the AluminiumSilver Alloys in the Guinier-Preston zones segregated state, we proposed a model based on the proximity effects between normal and superconductive phases. The results are in agreement with the hypothesis of the existence of a metastable miscibility gap governing the zone growth.
\end{abstract}

Dans le cadre de l'étude des relations entre les propriétés supraconductrices et les transformations structurales dans les alliages, nous présentons ici des résultats relatifs à des alliages Aluminium-Argent (riches en Aluminium) dans l'état de ségrégation (ou pré-précipitation). La solution solide sursaturée d'Argent dans l'aluminium se décompose durant un vieillissement

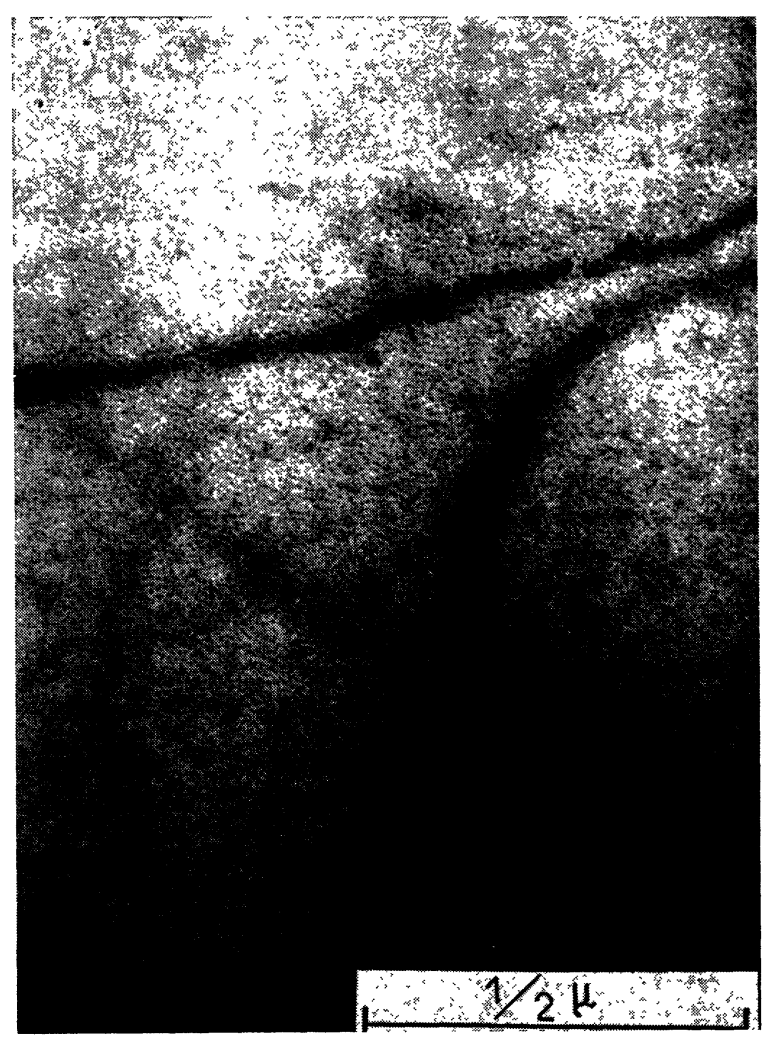

Fig. 1. - Al 5,02\% At Ag, homogénéisé 24 h à $550^{\circ} \mathrm{C}$, trempé et vieilli 9 heures à $140{ }^{\circ} \mathrm{C}$. isotherme selon la séquence suivante : zones GuinierPreston (Fig. 1) $\rightarrow$ précipitation de la phase $\gamma^{\prime}$ constituée de plaquettes riches en Argent (Fig. 2) $\rightarrow$ précipitation de la phase $\gamma$ d'équilibre de concentration $\simeq \mathrm{Ag}_{2} \mathrm{Al}$ [1]. Les zones Guinier-Preston sont sphériques, riches en Argent ( $\simeq 60 \% \mathrm{At}$ ), de même struc-

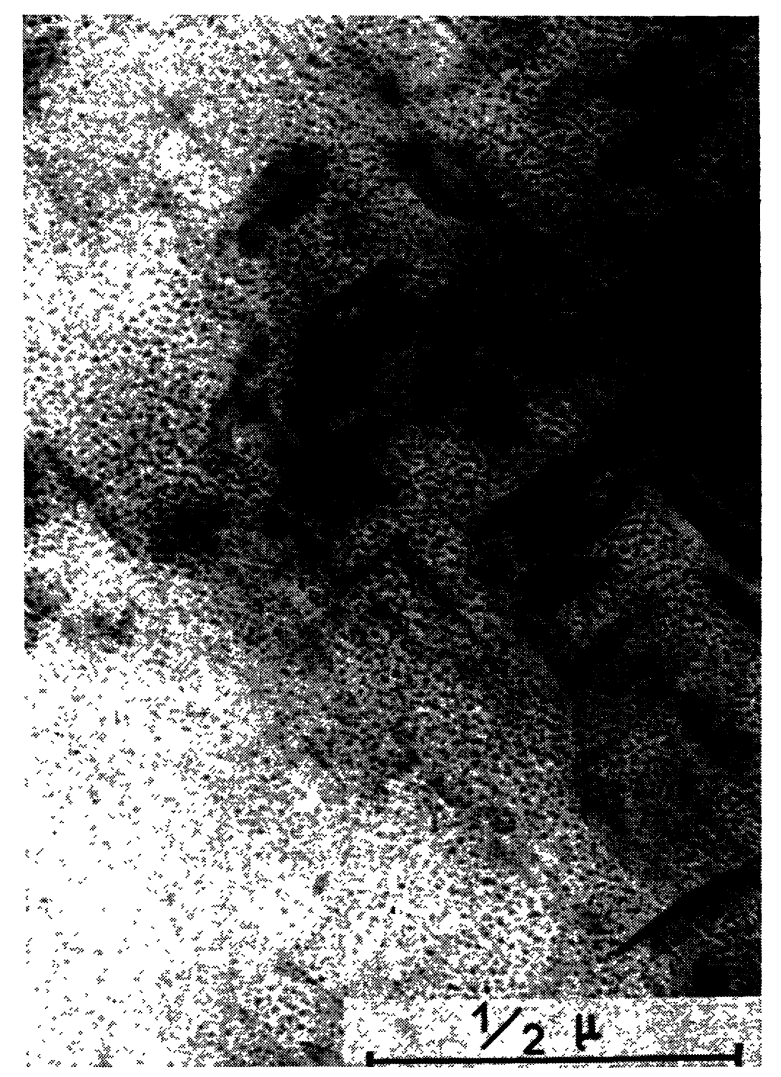

Fig. 2. - Al 5,02\% At Ag, homogénéisé 24 h à $550^{\circ} \mathrm{C}$, trempé et vieilli 8 jours à $140^{\circ} \mathrm{C}$. 
ture cristallographique que la matrice et cohérentes avec elle. Les alliages de concentrations 4,15\%,5,02\%, $6,7 \%, 10,10 \%$ At en Argent nous ont été fournis par la Société Péchiney-Soferec sous forme de bandes de $0,2 \mathrm{~mm}$ d'épaisseur. Ils ont été homogénéisés pendant 24 heures à $550^{\circ} \mathrm{C}$ sous atmosphère d'Argon, et trempés à l'eau. Ils ont ensuite été vieillis dans un bain

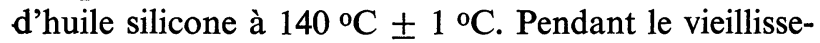
ment, les zones croissent et nous avons mesuré le diamètre moyen ainsi que la distance moyenne entre zones, par microscopie électronique en transmission et diffusion aux petits angles $(*)$ (Tableau I). La résistivité résiduelle (Fig. 3), $\rho_{0}$, mesurée à $4,2{ }^{\circ} \mathrm{K}$ décroît au cours du temps. Le changement de pente vers 24 heures pour Al-5,02\% At Ag coïncident avec l'apparition de premières plaquettes de la phase $\gamma^{\prime}$.

\section{TABleaU I}

$\mathrm{Al} 5,02 \%$ At $\mathrm{Ag}$, diamètre des zones mesuré par microscope électronique $d_{\mathrm{M}}$ et diffusion aux petits angles $d_{\mathrm{D}}$ et distance moyenne entre zones.

\begin{tabular}{|c|c|c|c|c|c|}
\hline $\begin{array}{l}\text { Temps de } \\
\text { vieillisse- } \\
\text { ment à } \\
140^{\circ} \mathrm{C}\end{array}$ & $2 \mathrm{~mm}$ & $6 \mathrm{~mm}$ & $20 \mathrm{~mm}$ & $1 \mathrm{~h}$ & $9 \mathrm{~h}$ \\
\hline$d \mathrm{M}(\bar{\AA})$ & $\overline{15 \AA}$ & $\overline{20 \AA}$ & $\overline{22 \AA}$ & $\overline{30 \AA}$ & $\overline{50 \AA}$ \\
\hline$d_{\mathbf{D}}(\AA ̊)$ & $22 \AA$ & $26 \AA$ & $29 \AA$ & $35 \AA$ & $55 \AA$ \\
\hline $\begin{array}{l}\text { listance } \\
\text { noyenne }\end{array}$ & IA & $74 \AA$ & $81 \AA$ & $106 \AA$ & $159 \AA$ \\
\hline
\end{tabular}

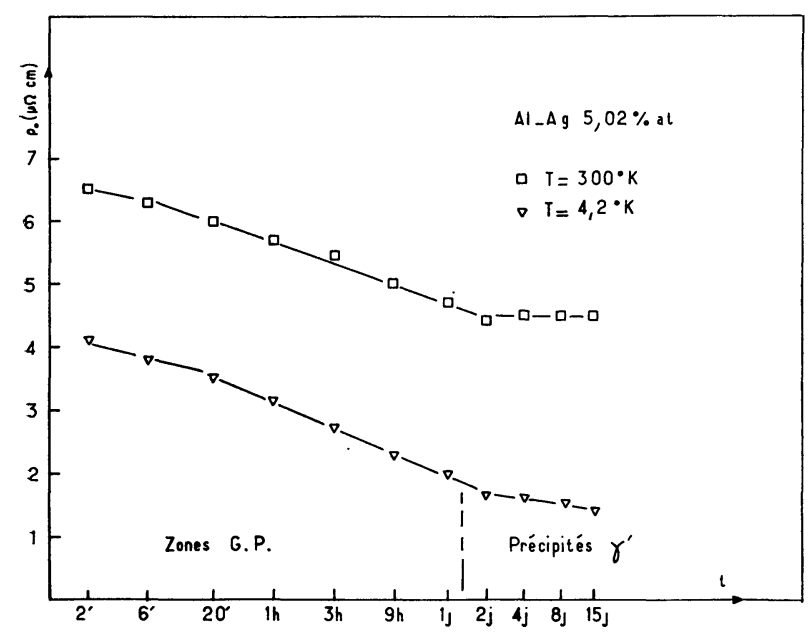

FIG. 3. - $\mathrm{Al} 5,02 \% \mathrm{At} \mathrm{Ag}$, variation de la résistivité, $\rho\left(300^{\circ} \mathrm{K}\right)$ et $\rho_{0}$ pendant le vieillissement.

Nous avons suivi l'évolution des propriétés supraconductrices par la mesure de cycles d'aimantation isothermes [2] et par transitions résistives. Les résultats,

(*) Les mesures de diffusion aux petits angles ont été effectuées au laboratoire de Métallurgie Physique de la Faculté des Sciences de Poitiers. rapportés à titre d'exemple pour l'alliage $\mathrm{Al}-\mathrm{Ag} 5 \% \mathrm{At}$, sont les suivants :

- Tant que les seules zones G. P. sont présentes, le comportement magnétique est quasi réversible et n'évolue pas, la température critique (Fig. 4) et le champ critique thermodynamique $H_{\mathrm{c}}$ restent constants alors que le champ critique $H_{\mathrm{c} 2}$ décroît linéairement avec $\rho_{0}$ (Fig. 5). La largeur de la transition résistive est étroite $(\simeq 0,02 \mathrm{~K})[3]$.

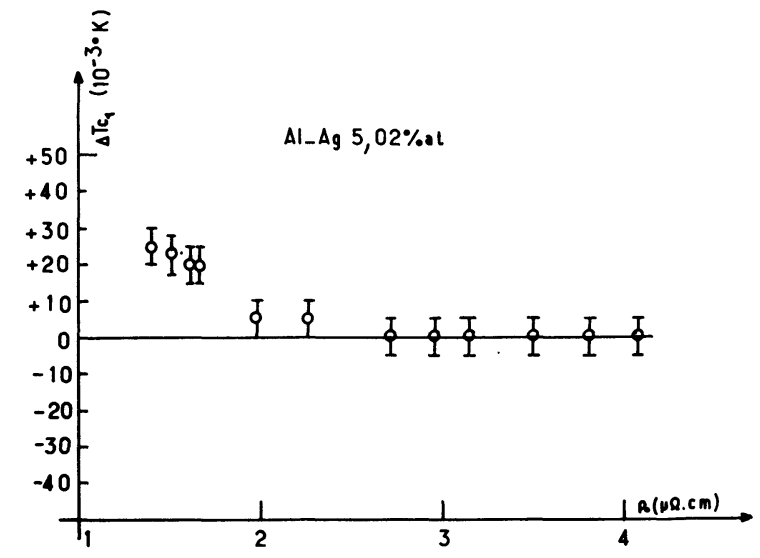

Fig. 4. - - Al 5,02\% At Ag, variation de $\Delta T_{\mathrm{c} 1}=T_{\mathrm{c}}$ (alliage vieilli, $T_{\mathrm{c}}$ (alliage trempé) avec $\rho_{0}$.

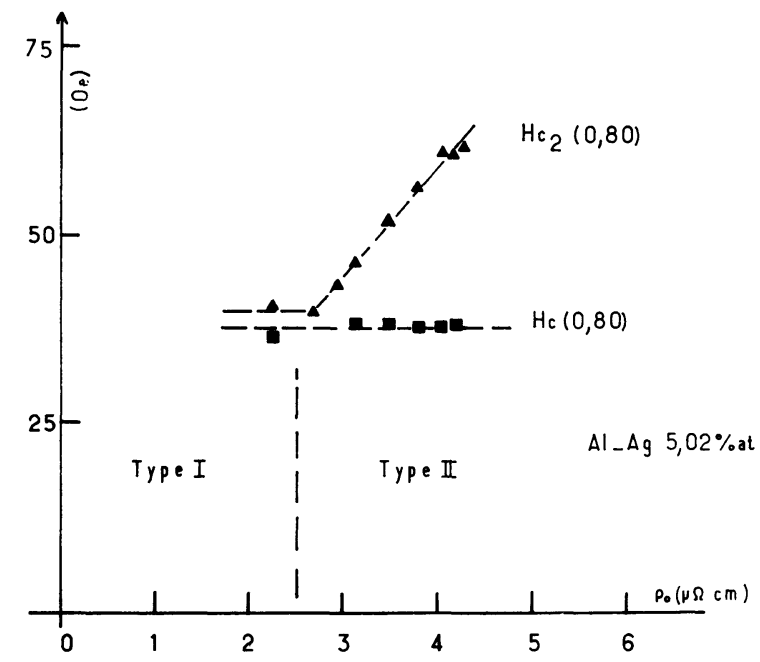

FIG. 5. - $\mathrm{Al} 5,02 \%$ At $\mathrm{Ag}$, variations des champs critiques $H_{\mathrm{c}}$ et $\bar{H}_{\mathrm{c} 2}$, à $T=0,80{ }^{\circ} \mathrm{K}$ pendant le vieillissement.

- Avec l'apparition du précipité $\gamma^{\prime}$, le comportement magnétique devient irréversible [4], la transition résistive s'élargit et la température critique augmente (Fig. 4).

Les résultats sont identiques pour les autres alliages et nous avons réuni en particulier, sur la figure 6 , toutes les valeurs mesurées de $T_{\mathrm{c}}$. On peut donc conclure que durant la pré-précipitation, les propriétés supraconductrices telles que la largeur de transition, la résistivité, $T_{\mathrm{c}}$ et $H_{\mathrm{c}}$, apparaissent insensibles à la présence 
d'inhomogénéités de concentration de l'ordre de $100 \AA$, échelle bien plus petite que la longueur de cohérence $\xi_{s} \simeq 2000 \AA$ aux températures utilisées.

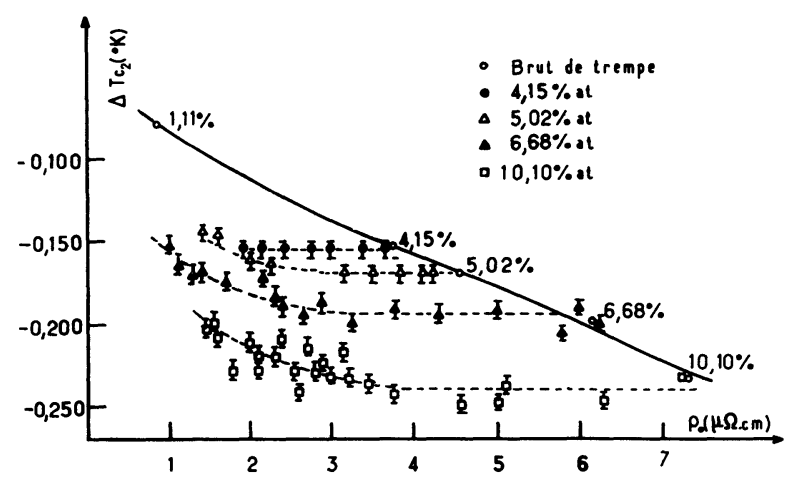

Fig. 6. - Températures de transition des alliages $\mathrm{Al}-\mathrm{Ag}$ brut de trempe et vieillis

$$
\Delta T_{\mathrm{c} 2}=T_{\mathrm{c}} \text { (alloy)- } T_{\mathrm{c}}(\mathrm{Al}), T_{\mathrm{c}}(\mathrm{Al})=1,175^{\circ} \mathrm{K} .
$$

Nous considérons maintenant la solution solide ségrégée comme un ensemble de lames normales ( $N=$ zones G. P) et supraconductrices $(S=$ matrice) en contact les unes avec les autres, d'épaisseur $d_{N}$ (diamètre de zones) et $d_{s}$ (distance moyenne). $d_{s}$ et $d_{N}$ sont respectivement plus petites que les longueurs de cohérence $\xi_{s}$ et $\xi_{N}$,

$$
\left[\xi_{s} \simeq 2000 \AA, \xi_{N}=\left(\frac{\hbar V_{F} l}{6 \pi K T}\right)^{1 / 2} \simeq 2500 \AA\right] .
$$

Nous sommes donc en présence d'un problème d'effet de proximité dans le cas de la limite de Cooper $[5,6]$. A chaque bi-lame $N-S$ correspond un paramètre $(N V)$ effectif calculé par de Gennes [7].

$$
(N V)_{\text {eff }}=\frac{V_{n} N_{n}^{2} d_{n}+V_{s} N_{s}^{2} d_{s}^{2}}{N_{n} d_{n}+N_{s} d_{s}}
$$

où $N_{n}, N_{s}$ sont les densités d'états, $V_{n}$ et $V_{s}$ les paramètres d'interaction électron-électron. Nous généralisons cette expression pour un ensemble de bi-lames à trois dimensions, d'où :

$$
(N V)_{\mathrm{eff}}=\frac{V_{n} N_{n}^{2} \beta+V_{s} N_{s}^{2}(1-\beta)}{N_{n} \beta+N_{s}(1-\beta)},
$$

$\beta$ étant la fraction de volume de métal normal. $T_{\mathrm{c}}$ étant constant pendant la pré-précipitation, et donné par l'expression B. C. S. $T_{\mathrm{c}} \simeq 0,85 \theta_{\mathrm{D}} \exp \left(-\frac{1}{(N V)_{\text {eff }}}\right)$, on en déduit que la croissance des zones doit se faire à fraction de volume de métal normal constant.

Ce mécanisme avait été supposé par des études métallurgiques sur la ségrégation dans ces alliages. Gerold et al. [8] par des mesures de diffusion aux petits angles ont établi qu'une lacune de miscibilité métastable contrôlait la formation de zones G. P. dans Al-Ag (Fig. 7). Toutefois, Bonfiglioli et Guinier [9] avaient précisé qu'une telle situation ne pouvait s'établir immédiatement après trempe, mais seulement dans un état final où les deux phases ont alors des concentra- tions bien définies. Dans notre cas, la trempe utilisée n'est pas très rapide et le diamètre minimal des zones mesurées nous laisse penser que nous sommes dans cet état final. Nous avons $m_{\mathrm{A}}=\beta m_{1}(T)+(1-\beta) m_{2}(T)$ où $m_{\mathrm{A}}$ est la concentration nominale en Argent, $m_{1}(T)$ et $m_{2}(T)$ la concentration en Argent respectivement des zones et de la matrice, $T$ la température de vieillissement. La fraction en volume $\beta$ ne dépend que de $m_{\mathrm{A}}$ et $T$.

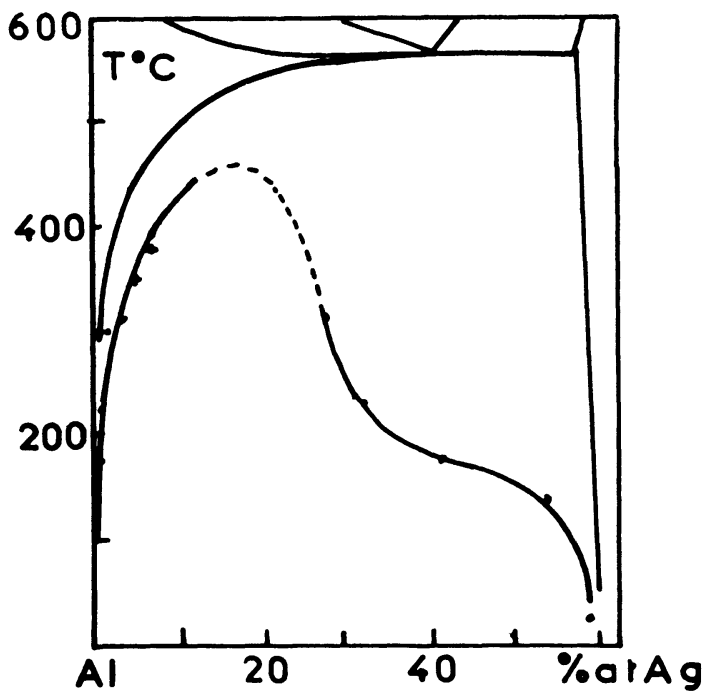

Fig. 7. - Diagramme d'équilibre du système $\mathrm{Al}-\mathrm{Ag}$ avec la lacune de miscibilité.

La solution solide ségrégée se comporte, alors, comme un supraconducteur « Sale » $\left(l<\xi_{0}\right)$ et homogène avec un paramètre $N V=(N V)_{\text {eff }}$ unique, ce qui explique une largeur de transition étroite et un comportement magnétique quasi réversible. La température $T_{\mathrm{c}}$ et le champ critique $H_{\mathrm{c}}$ (reliés à $(N V)_{\text {eff }}$ par l'expression de l'énergie de condensation [10]) sont constants durant la pré-précipitation, mais varient avec la concentration $m_{\mathrm{A}}$ de l'alliage. A cause de leur dépendance avec le libre parcours moyen, $H_{\mathrm{c} 2}$ et $H_{\mathrm{c} 3}$ (mesuré à partir des courbes d'aimantation avec le champ magnétique parallèle à la surface de l'échantillon), décroissent durant le vieillissement et leur variation est en accord avec la relation de Gorkov-Goodman (Fig. 5) [11] et les résultats sur la supraconductivité de surface (Fig. 8) [12].

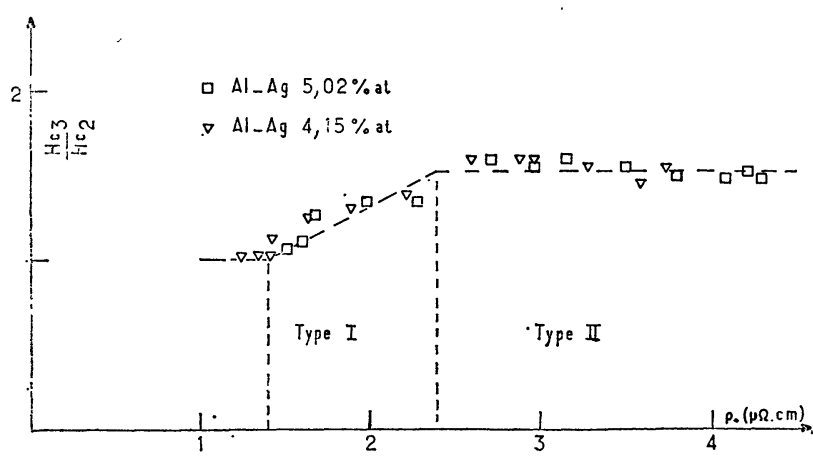

FIG. 8. - Variation du champ $H_{\mathrm{c} 3}$ au cours du vieillissement. 
Nous évaluons la température de transition pour différents alliages à l'aide de l'expression B. C. S. où la variation de $\theta_{\mathrm{D}}$ est négligée. On prend

$$
\frac{N_{\mathrm{s}}}{N_{n}}=\frac{\gamma_{\mathrm{Al}}}{\gamma_{\mathrm{Ag}}}=2,08,
$$

$m_{1}\left(140{ }^{\circ} \mathrm{C}\right)=53,9 \%$ At et $m_{2}\left(140^{\circ} \mathrm{C}\right)=0,71 \%$ At [6], et nous calculons la différence $\Delta T_{\mathrm{c}}=T_{\mathrm{c}}-T_{\mathrm{c}}^{0}$, où $T_{\mathrm{c}}$ est la température de transition de l'alliage de concentration $m_{\mathrm{A}}$ et $T_{\mathrm{c}}^{0}$ celle de l'alliage pour lequel $\beta=0\left(m_{\mathrm{A}} \# 0,71 \% \mathrm{At}, T_{\mathrm{c}}^{0}(0,71 \% \mathrm{At})=1,12 \mathrm{~K}\right)$. Sur la figure 9, l'écart entre les deux courbes calculées montre une forte dépendance de $\Delta T_{\mathrm{c}}$ avec $(N V)_{n}$. Le meilleur accord est obtenu pour $(N V)_{n}=0,1$. Ce résultat est à rapprocher de la valeur $(N V)_{\mathrm{Ag}}=0,1$ obtenue par Deutscher et al. [14].

L'écart entre la courbe théorique et les points expérimentaux peut avoir au moins deux causes. La frontière entre les phases $N$ et $S$ n'est probablement pas définie à mieux qu'une ou deux distances interatomiques, néanmoins c'est une frontière propre sans la présence d'oxyde qui limiterait sérieusement l'application de la théorie de la limite de Cooper. Nous avons enfin négligé l'influence de l'Argent sur le spectre de phonons de l'Aluminium et considéré que l'effet principal était de nature électronique et dû à la proximité d'un métal normal et d'un métal supraconducteur.

Les mesures des propriétés supraconductrices du système $\mathrm{Al}-\mathrm{Ag}$ et leur interprétation accrédite l'hypothèse de l'existence d'une lacune de miscibilité proposée

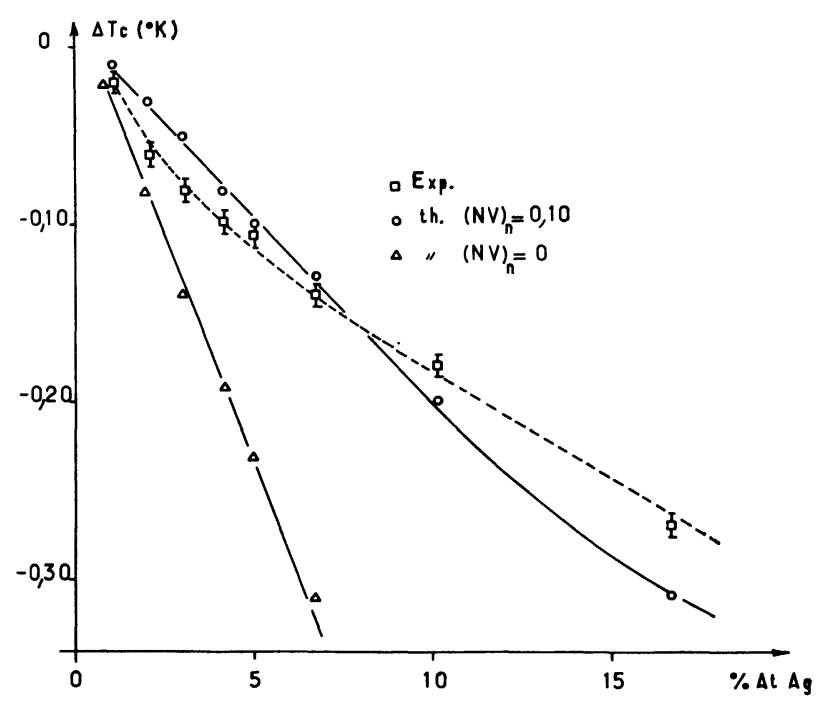

Fig. 9. - Variation expérimentale et théorique de $T_{\mathrm{c}}$ avec la concentration en $\mathrm{Ag}$.

$$
\Delta T_{\mathrm{c}}=T_{\mathrm{c}}(\text { alliage } \beta \neq 0)-T_{\mathrm{c}}^{0}(\text { alliage } \beta=0) .
$$

par Gerold. Ce résultat montre aussi que la supraconductivité peut être utilisée comme moyen supplémentaire pour l'étude de certains systèmes métallurgiques.

Remerciements. - Nous tenons à remercier M. Bethoux et M. Wintenberger pour de nombreuses et utiles discussions ainsi que $\mathbf{M}$. Geneste qui a réalisé les observations au microscope électronique. Ce travail a été réalisé avec l'aide de la D. G. R. S. T.

\section{Bibliographie}

[1] Walker (C. B.) and Guinier (A.), Acta Met., 1953, 1, 568.

Nicholson (R. B.) and Kelly (A.), Precipitation hardening, p. 255, Progress in material sciences 10. Edit. Chalmers.

[2] Nemoz (A.), Solecki (J. C.), Faure (J. P.) et Keyston (J. R. G.), Rev. Phys. Appl., 1969, 4, 42.

[3] Solecki (J. C.), Thèse $3^{\mathrm{e}}$ cycle, nov. 1969.

[4] Blanc (J.), Goodman (B. B.) and Nemoz (A.), Proceedings of the Xth international Conference on Low Temperature Physics, Moscow, 1966.

Blanc (J.), Geneste (J.) and Nemoz (A.), Proceedings of the Colloquium of Bakouriani, Georgia (U. S. S. R.), 1968.

[5] CoOPer (L.), Phys. Rev. Letters, 1961, 6, 698.

[6] Deutscher (G.) and De Gennes (P. G.), Superconduc- tivity, Edited by R. D. Parks (Marcel Dekker, Inc. N. Y. 1969, Vol. 2, Chap. 17).

[7] De Gennes (P. G.), Rev. Mod. Phys., 1964, 36, 225.

[8] Baur (R.) and Gerold (V.), Acta Met., 1962, 10, 637.

[9] Bonfiglioli (A. T.) and Guinier (A.), Acta Met., 1966, 14, 1213.

[10] De GenNes (P. G.), Superconductivity of metals and alloys (W. A. Benjamin, Inc. N. Y., 1966).

[11] Goodman (B. B.), I. B. M. Journal, 1962, 6, 63.

[12] Saint-James (S.) and De Gennes (P. G.), Phys. Letters, $1963,7,306$.

[13] Kittel (C.), Introduction to Solid State Physics (John Wiley and Sons, Inc. N. Y., 1966).

[14] Deutscher (G.), Lindenfeld (P.) and Wolf (S.), Phys. Rev. Letters, 1969, 23, 1102. 